\title{
Boom Minimization Framework for Supersonic Aircraft Using CFD Analysis
}

\author{
Irian Ordaz* \\ NASA Langley Research Center, Hampton, VA 23681 \\ Sriram K. Rallabhandi ${ }^{\dagger}$ \\ National Institute of Aerospace, Hampton, VA 23666
}

\begin{abstract}
A new framework is presented for shape optimization using analytical shape functions and high-fidelity computational fluid dynamics (CFD) via Cart3D. The focus of the paper is the system-level integration of several key enabling analysis tools and automation methods to perform shape optimization and reduce sonic boom footprint. A boom mitigation case study subject to performance, stability and geometrical requirements is presented to demonstrate a subset of the capabilities of the framework. Lastly, a design space exploration is carried out to assess the key parameters and constraints driving the design.
\end{abstract}

\section{Nomenclature}

Acronyms

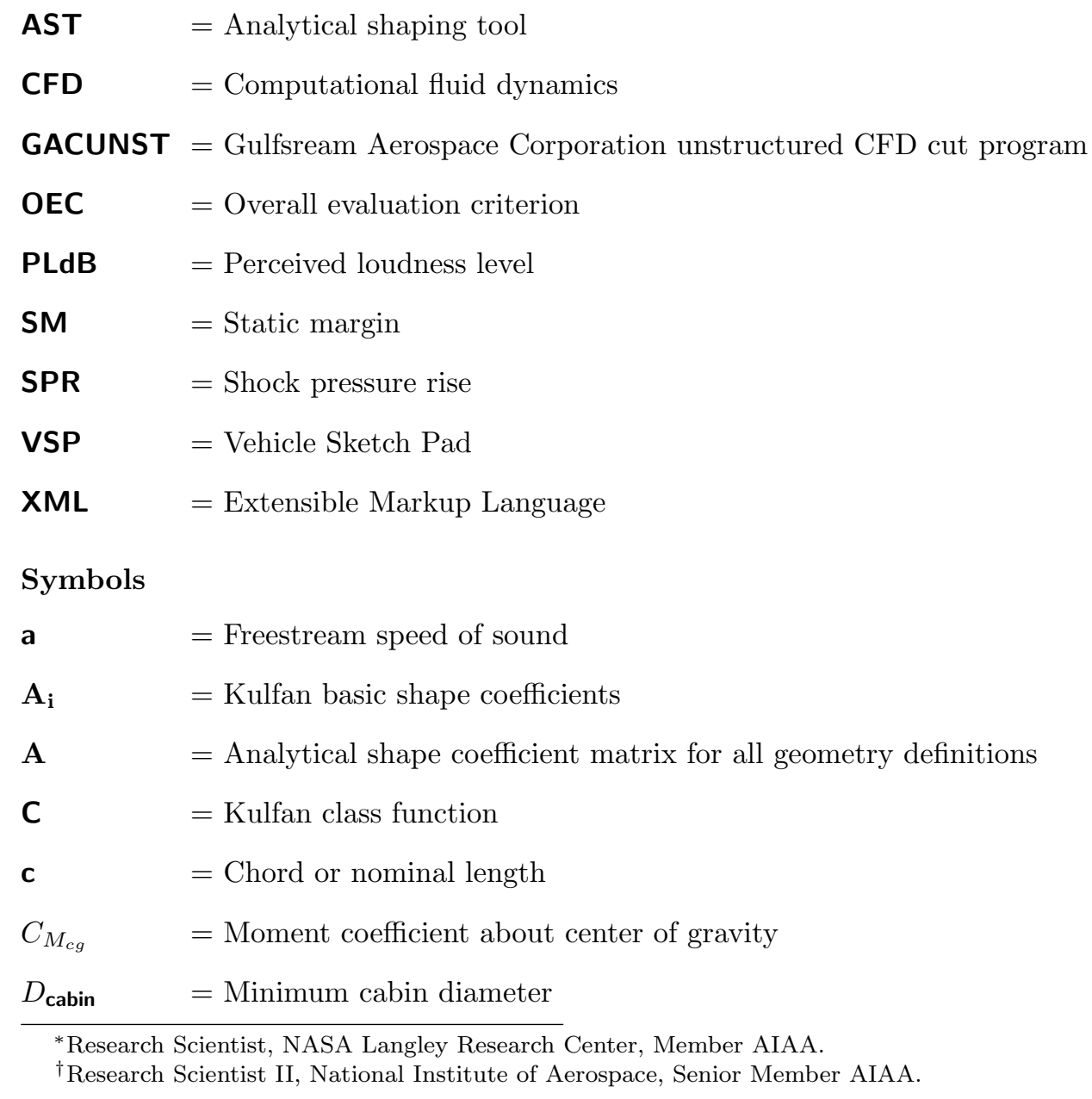




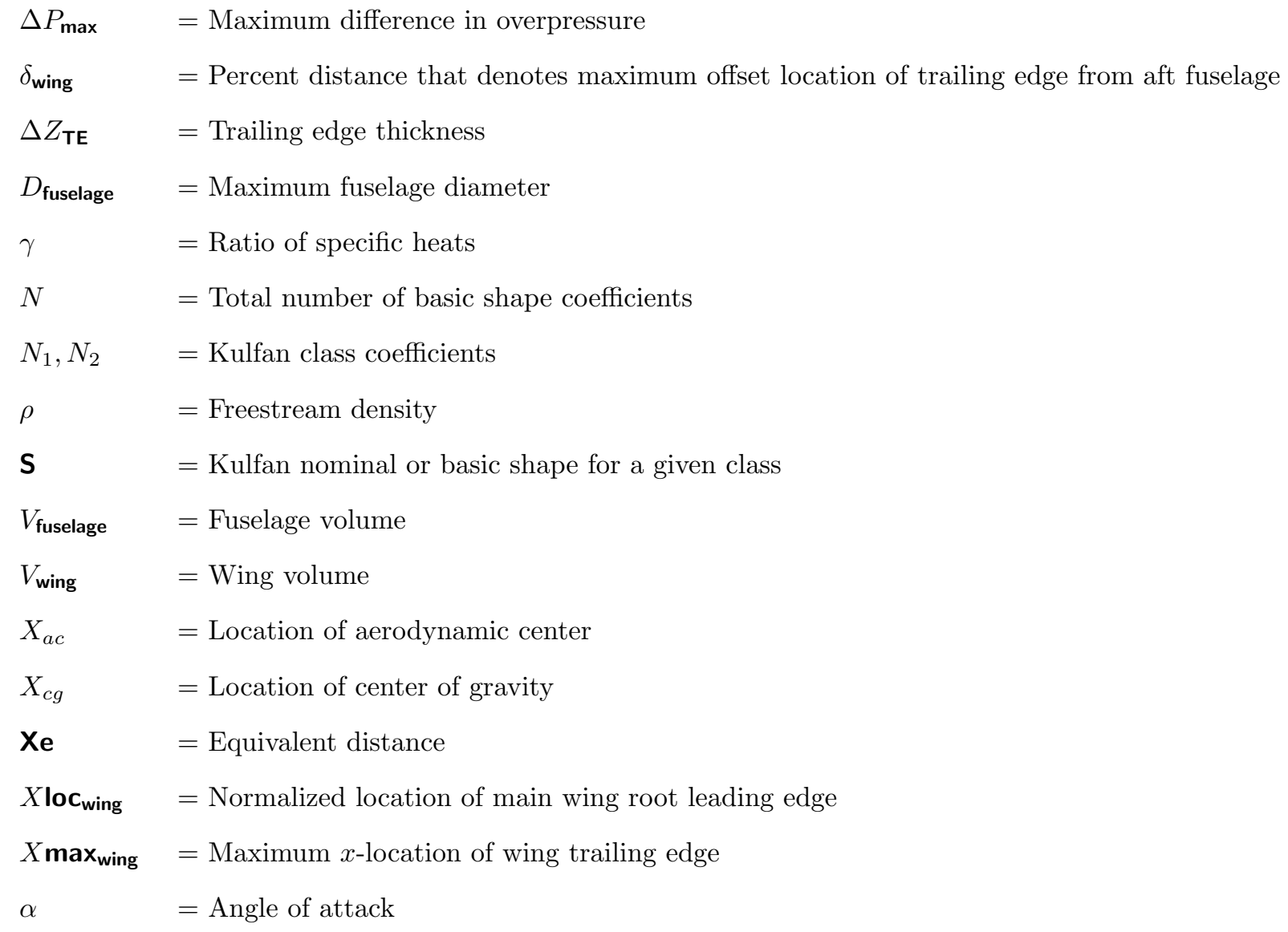

\section{Introduction}

DESGN of a low boom aircraft remains a challenging problem given the contradictory trends that exist between design for low sonic boom and improved performance. Low sonic boom design is complicated because it intrinsically connects all components into a complex interaction problem for which local tailoring of the aircraft shape is unlikely to significantly improve the low-boom characteristics of the vehicle. This paper demonstrates an automated Model Center ${ }^{1}$ framework that handles the abovementioned objectives by using analytical shape functions to represent the surface definition of the vehicle and state-of-the-art computational fluid dynamics (CFD) techniques. The following sections describe the intended analysis path and tools, as well as the problem formulation.

\section{Background}

This section introduces several key modeling techniques and the tools that were used in the current formulation, including shape parameterization, aerodynamic analysis, relevant sonic boom theory, and sonic boom propagation.

\section{A. Shape Function-Based Geometry Definition}

The mathematical representation of aircraft components, such as airfoils and fuselages, is generally continuous but nonanalytic as a result of the existing infinite slope at the pointed trailing edges and a higher degree of curvature near the leading edges. ${ }^{2}$ As a result, definition of the aforementioned geometry components requires a large number of control point coordinates. Kulfan and Bussoletti ${ }^{2}$ introduce a simple analytical shape function that is capable of handling such geometrical requirements. The general form of the shape function is given in Eq. (1a). ${ }^{2}$ The class function (C) given in Eq. (1b) can be manipulated using the two class function coefficients $N_{1}$ and $N_{2}$. The basic shape of the geometry is controlled using the function $\mathrm{S}$ given in Eq. (1c) consisting of the coefficients $\mathbf{A}_{\mathbf{i}}$. This function, if set to one becomes the nominal shape for a given class function. The function also allows direct control of both the leading edge radius and the trailing edge boattail angle and closure to a specified aft thickness $\left(\Delta Z_{\mathrm{TE}}\right)$. 


$$
\begin{aligned}
\frac{z}{c} & =(\mathrm{C})(\mathrm{S})+\left(\frac{x}{c}\right) \frac{\Delta Z_{\mathrm{TE}}}{c} \\
\mathrm{C} & =\left(\frac{x}{c}\right)^{N_{1}}\left(1-\frac{x}{c}\right)^{N_{2}} \\
\mathrm{~S} & =\sum_{i=0}^{N}\left[A_{i}\left[\frac{x}{c}\right]^{i}\right]
\end{aligned}
$$

In the presented shape formulation, Kulfan ${ }^{2}$ and Bussoletti's shape functions are used to obtain the geometry definitions, such as the fuselage height, width, and airfoil cross sections. The work of Lores et al., ${ }^{3}$ along with other simple piecewise linear shape functions, is used to define the geometry parameters, such as camber, wing chord length, leading edge sweep, dihedral, twist, and airfoil thickness distributions. The chord definition can be defined by using either a strictly nonlinear or piecewise linear approach, or by using a combination of the two.

The use of shape functions permits geometrical flexibility by allowing the description of geometrical definitions to be defined through analytical functions. These analytical functions contain several coefficients that define the behavior and the shape of the function. Figure 1 shows a graphical representation of six shape functions that contribute to a thickness distribution; the black line depicts the mean shape. A low number of shape functions can lead to a more rigid geometry, which reduces the feasible design space in which the optimizer can search for an optimal solution. Conversely, a larger number of shape functions leads to a greater number of design variables for the optimizer, which increases the computational effort and the time requirements.

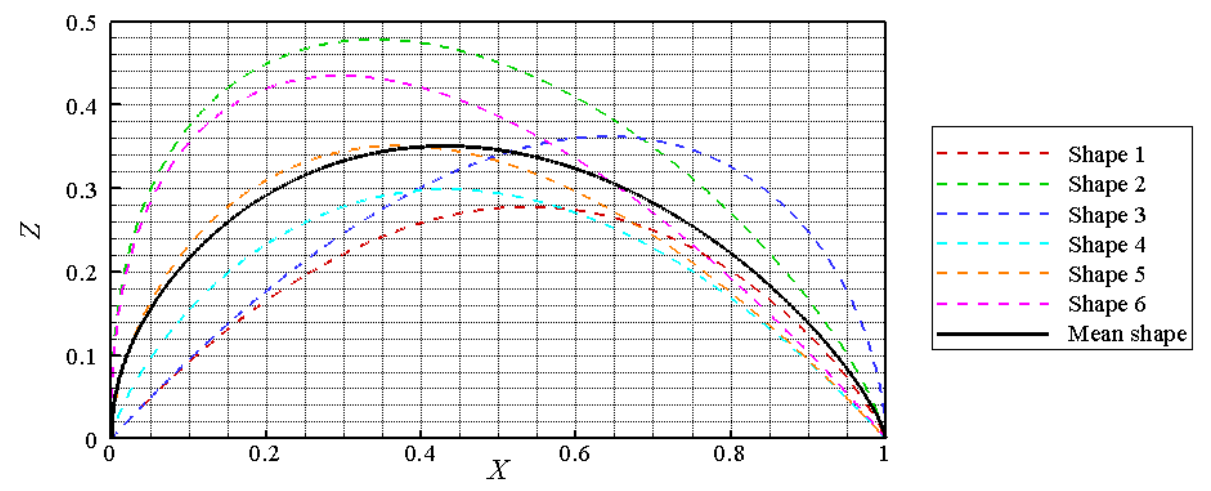

Figure 1. Shape function contribution to thickness distribution.

For better scaling, the shape parameterization is formulated in a manner such that all shape function coefficients, component locations along the fuselage axis, and spanwise stations are scaled between zero and one. One shape parameterization code, called Analytical Shaping Tool (AST), allows the user to perform the optimization for not only all of the aforementioned geometry definitions for the main wings, but also for additional components, such as canards, vertical, horizontal, and T-tails. The AST code also permits control of all component locations with respect to the fuselage. The AST program, coupled with high-fidelity CFD, facilitates system-level studies in the assessment of different vehicle configurations.

\section{B. Cartesian-Based CFD Using Cart3D}

Cart3D was the selected aerodynamics analysis tool for the optimization framework because it allows the exploration of nonconventional and complex designs for which low-fidelity analyses become unreliable. Cart3D is a high-fidelity inviscid analysis CFD package that is geared toward conceptual and preliminary aerodynamics design. ${ }^{4}$ The package allows for quick and automated Cartesian-based mesh generation and CFD solutions for complex geometries. It includes numerous tools that facilitate the conversion and import of various geometry formats. In addition, it is highly scalable and can be run on multiprocessor systems.

Another useful feature that makes Cart3D attractive is the capability to easily control the type of output data. Cart3D can export plane cuts for flow parameters at arbitrary locations, as well as line data files for pressures at off-body locations. A sample pressure solution that was obtained by using Cart3D $(\sim 2.5$ million mesh cells) is shown in Fig. 2 for a supersonic vehicle with flow-through nacelles at Mach 
1.8 and an angle of attack of $2.53 \mathrm{deg}$. Note that pressure in this paper is nondimensionalized by the reference quantity $\rho * a^{2}$ (freestream pressure $=1 / \gamma$ ). All values of the lift and drag forces that are given correspond to total lift and drag over the entire vehicle. These forces are normalized similar to pressure and for a unit area.

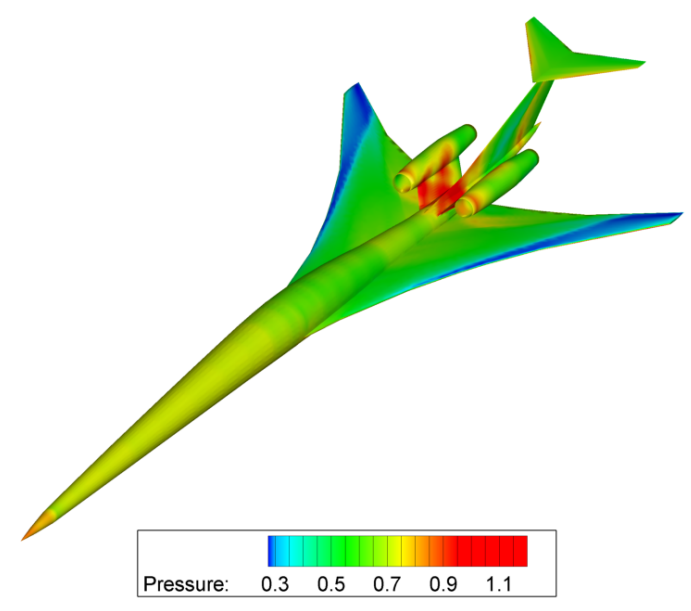

Figure 2. Cart3D surface pressure solution.

A Cart3D Model Center wrapper was developed as shown in Fig. 3 to integrate Cart3D into the framework. The wrapper provides the user with control over numerous inputs that range from the number of processors (shared or nonshared systems), flight conditions, refinement levels, the coefficient of lift design/matching, as well as the ability to extract line and point sensors and planar and cylindrical cuts.

\begin{tabular}{|c|c|}
\hline & \begin{tabular}{|l} 
Name \\
$\square \cdots$ Model \\
$-\cdots R T 3 D$
\end{tabular} \\
\hline 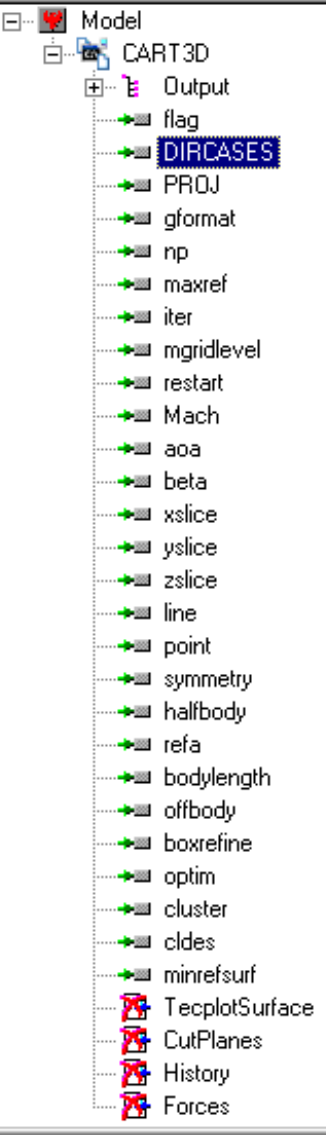 & $\begin{array}{l}0 \\
\text { /scratch } \\
\text { geometry } \\
\text { stl } \\
12 \\
11 \\
200 \\
3 \\
\text { no } \\
1.8 \\
2.53 \\
0 \\
\text { no } \\
0 \\
\text { no } \\
\text { no } \\
\text { no } \\
\text { yes } \\
\text { no } \\
1 \\
170 \\
1 \\
10 \\
\text { no } \\
\text { yes } \\
\text { no } \\
10 \\
\langle\text { view...〉 } \\
\langle\text { view... } \\
\langle\text { view... } \\
\langle\text { view... }\end{array}$ \\
\hline
\end{tabular}

Figure 3. Model Center wrapper for Cart3D.

The automated CFD solution process with Cart3D begins with shape function coefficients that gen- 
erate an XML geometry file for Vehicle Sketch Pad (VSP). ${ }^{5}$ The VSP geometry is then triangulated by using VSP's compgeom feature to produce a watertight stereo-lithography surface, as shown in Fig. 4 for the baseline vehicle. The stereo-lithography geometry is then automatically exported and used to generate a Cart3D CFD solution, as demonstrated in Fig. 4(c) which shows the pressure contours.

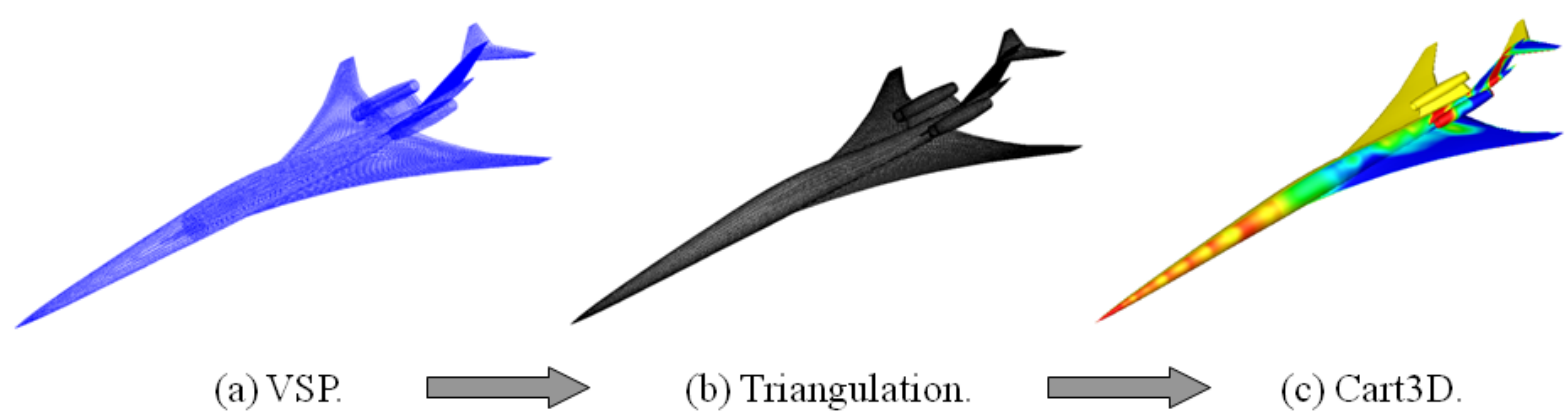

Figure 4. Automated CFD solution process.

\section{Sonic Boom Analysis}

Sonic boom prediction has been extensively studied and documented in the literature. ${ }^{6-11}$ For this study, we are particularly interested in extracting a mid-field form of the aircraft disturbance field and propagating it to the ground level to obtain the boom footprint. Several approaches are available for obtaining the mid-field distribution. The first method is to compute the surface pressures by using a CFD solver and then use numerical integration along the Mach plane cuts to generate equivalent area distributions. ${ }^{12}$ The second method is to extract a CFD pressure off-body cylinder and apply multipole methods ${ }^{13-15}$ to map the near-field pressures to their corresponding mid-field values so that the three dimensional cross-flow effects are taken care of before propagation to the ground. In the third approach, a CFD pressure solution that is obtained at several body lengths below the aircraft can be directly used for propagation to the ground. The downside in this third case is the relatively high computational cost that is associated with grid stretching and adaptation for resolving shocks far away from the aircraft. In the above approaches, the CFD run time successively increases from the first method, which requires only a surface solution, to the third approach, for which a very fine mesh is needed for locations that are far below the aircraft. Each approach has distinctive benefits, as well as characteristic pitfalls. Because this is a preliminary study for evaluating the performance of shape functions in low-boom design, the first approach was selected for the sonic boom analysis. The second and third approaches were considered to be too time consuming and perhaps inappropriate for conceptual optimization studies.

In the first approach, Cart3D is used to generate a surface pressure, as shown in Fig. 2. Then, integration is performed to obtain the corresponding equivalent area distributions (see Fig 5). Figure 5 also provides comparison with low-fidelity methods such as PBOOM. ${ }^{16}$ Discrepancies are obvious in the areas that are computed from the two methods. Although these discrepancies appear small, their impact is significant, as can be seen in Fig. 6. These ground signatures are obtained through propagation of equivalent areas to the ground by using ARAP. ${ }^{17}$ ARAP is a propagation code with fidelity similar to that of the more widely used PCBOOM. ${ }^{18}$ Both methods employ linear geometric acoustics and weak shock theory to propagate pressure distributions to the ground. To study the sonic boom impact, the most widely accepted metrics (e.g. perceived loudness and overpressure levels) are used as measures of low-boom characteristics. 


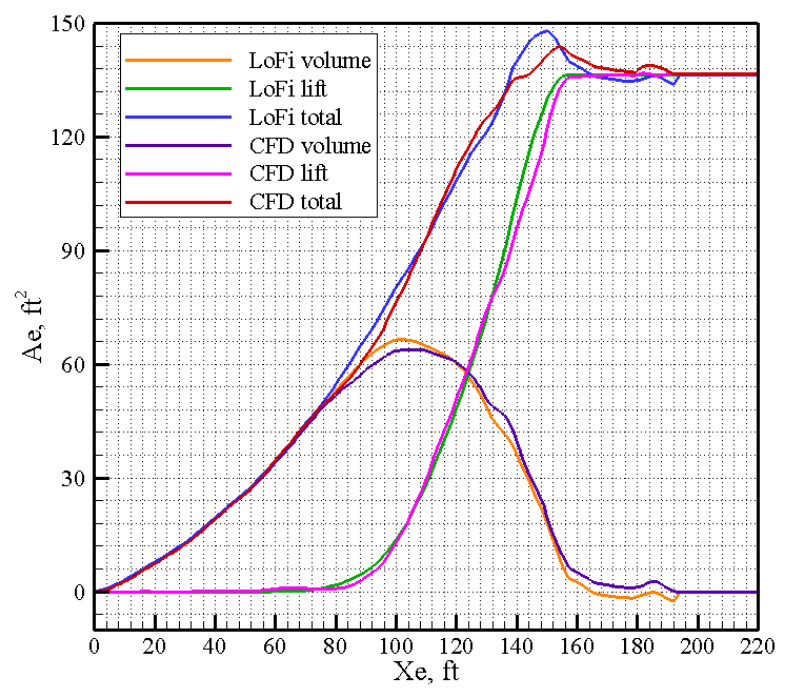

Figure 5. High- and low-fidelity equivalent areas.

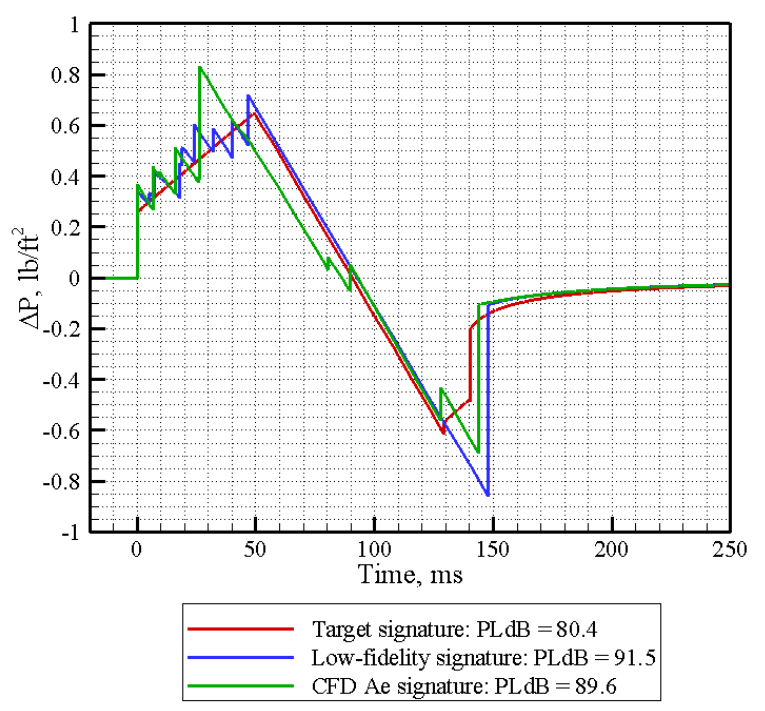

Figure 6. Comparison of high- and low-fidelity ground signatures.

\section{Problem Setup}

The design problem, the analysis processes, and the optimization framework are described in this section. In addition, a case study is presented to demonstrate a subset of the framework capabilities.

\section{A. Problem Statement and Approach}

This paper demonstrates an automated framework for boom minimization that uses analytical shape functions for a supersonic vehicle. The framework allows for the optimization to take place on a global scale but also allows the user to focus the optimization on specific geometrical definitions of a baseline vehicle. This means that optimization can be performed globally on the entire geometry or focused to specific parameters, such as fuselage radius, wing chord length, and leading edge sweep. The cost of global optimization is an increasing number of design variables, which can make a problem unmanageable.

The Model Center framework developed for the design optimization is shown in Fig. 7, and the corresponding analysis tools are given in Table 1. Model Center's Design Explorer ${ }^{1}$ was chosen as the 
optimizer because it provides a compromise in robustness and efficiency between gradient-based optimizers and fully stochastic methods, such as genetic algorithms. Design Explorer is based on a surrogate-model method for constraint optimization. ${ }^{19}$ The surrogate modeling technique that is implemented within Design Explorer is a Kriging model. ${ }^{20,21}$

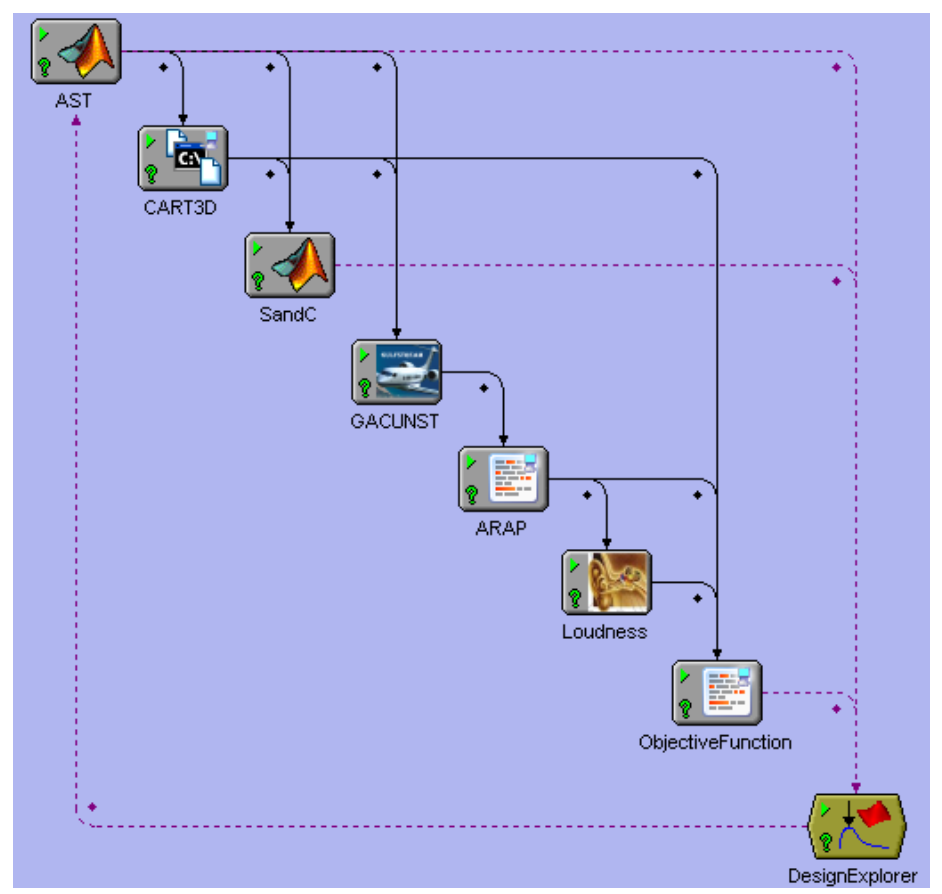

Figure 7. Design and optimization framework.

Table 1. Analysis Tools.

\begin{tabular}{ll}
\hline Analysis & Tool/theory \\
\hline \hline Shaping & AST \\
Geometry & VSP \\
CFD & Cart3D \\
Equivalent area & GACUNST \\
Propagation & ARAP \\
Stability & Static margin \\
\hline
\end{tabular}

The first step in the design process requires a decision on the type of geometrical definitions of interest. These definitions could include the fuselage radius, wing sweep, chord, and so on. If optimization is to be carried out only on specific definitions, then the additional information must be provided for the remaining definitions to fully describe the vehicle. This missing information can be input in two different ways. One option is to explicitly provide the shape function coefficients, locations, and other pertinent information as direct inputs to the AST. The second, and more practical option is to make a baseline VSP geometry available for AST to extract the missing information.

In the presented case study, optimization is performed on the fuselage radius, wing chord, and sweep spanwise distributions, as well as wing's $x$-location and root chord length. The fuselage length and wing span are fixed at $170 \mathrm{ft}$, and $57.76 \mathrm{ft}$, respectively. The cabin length is set to 25 percent of the length of the fuselage, and a diameter of at least $7 \mathrm{ft}$. The fuselage camber, tail, pylon and nacelle configurations are not permitted to vary from that of the baseline. This fixed geometry information is imported directly from the baseline vehicle that is shown in Fig. 8. The baseline vehicle referenced in this paper is the result of extensive work and manual design iterations by design and boom experts. 

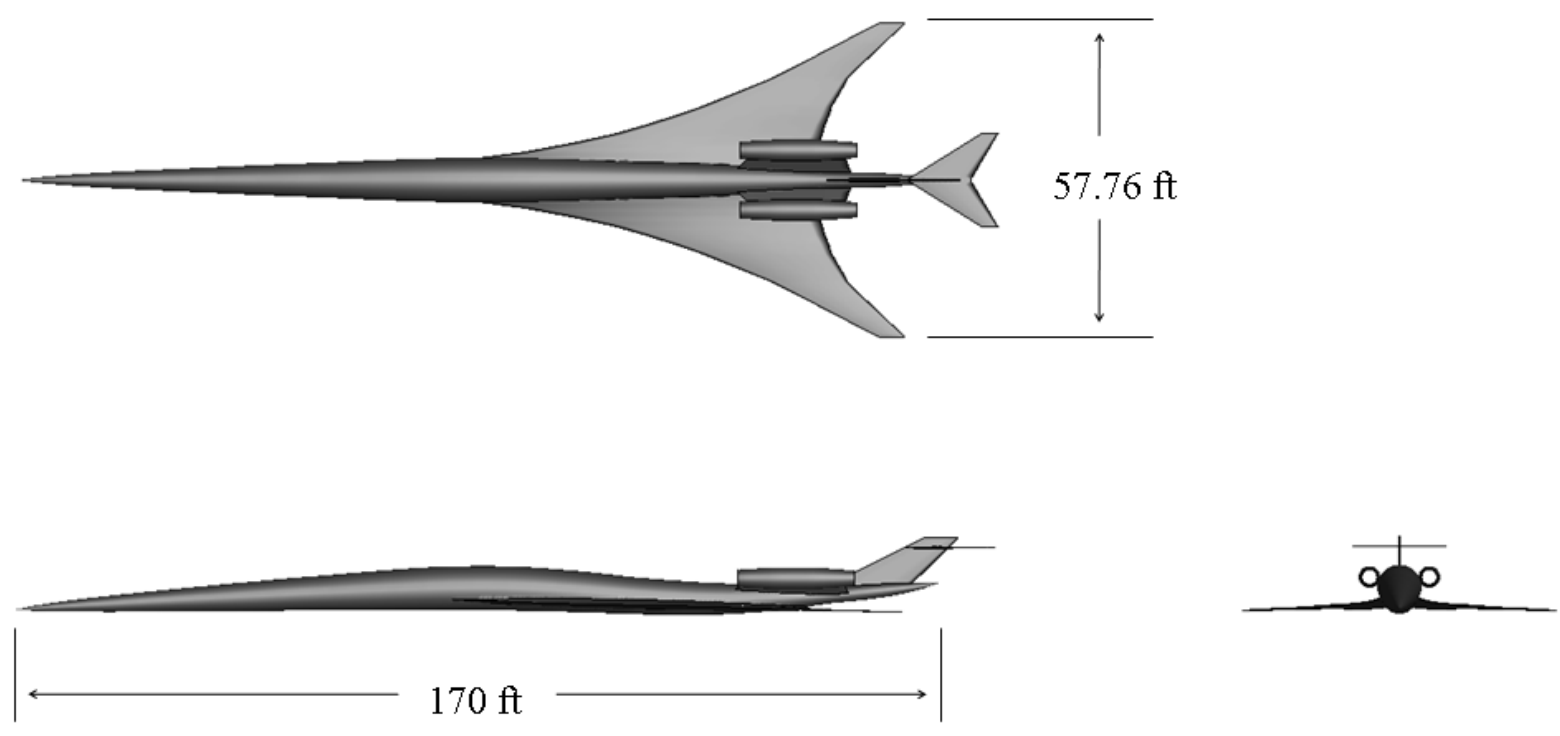

Figure 8. Orthographical views of baseline vehicle.

Once the basic geometrical definitions have been generated by AST, a VSP geometry is created, and the outer mold line, including surface triangulation, is exported by using VSP in stereo-lithography format. This mold line and triangulation definition is then imported into Cart3D to compute a CFD solution. The CFD mesh that is generated during the optimization is between 1 and 1.5 million cells in size. The mesh size was chosen with the goal of balancing computational cost and accuracy; mesh sensitivity study was performed to better understand this tradeoff with regard to aerodynamic and sonic boom analysis. The results of this mesh sensitivity study are provided in appendix A. The study demonstrates that a grid size of approximately one million cells is sufficient to predict aerodynamic and sonic boom characteristics reasonably well.

The CFD-based inviscid surface pressure distribution is used with Gulfstream's GACUNST ${ }^{12}$ code to compute the lift, volume, and total equivalent area distributions. The resulting equivalent areas are used by ARAP to compute the ground signatures. The ground signature is computed based on an assumption of steady level flight at an altitude of 51,650 ft with no atmospheric wind. Finally, the ground signature is input into a loudness code ${ }^{22}$ to obtain the perceived noise levels (PLdB).

The stability and control analysis component in Model Center is based on simple longitudinal static stability theory (i.e. pitching behavior about the $Y$-axis). This requires the estimation of both the center of gravity $\left(X_{c g}\right)$ with VSP and the aerodynamic center $\left(X_{a c}\right)$ based on the Cart3D surface pressures. The vehicle is considered to be statically stable in the longitudinal dimension if it satisfies Eq. (2). Note that that the VSP center of gravity is a crude estimation because VSP is a conceptual geometry package that accounts only for the outer mold line of the vehicle. Although internal components can be placed in VSP, the code does not adequately handle detailed internal layout design, which can impact the center of gravity of the aircraft.

$$
\begin{gathered}
\text { Static margin }(\mathrm{SM})=\frac{\partial C_{M_{c g}}}{\partial \alpha}=-\left(\frac{X_{a c}-X_{c g}}{c}\right)<0 \\
X_{a c}>X_{c g}
\end{gathered}
$$

\section{B. Design Objectives and Constraints}

A numerical optimizer is implemented to drive an objective function, which can be a single value or a weighted overall evaluation criterion (OEC). For the present case study, a single objective is utilized to simplify the problem. The vehicle's total drag is used to quantify aerodynamic efficiency for a given baseline lift value. The perceived loudness level $(\mathrm{PLdB})$ and the maximum change in overpressure $\left(\Delta P_{\max }\right)$ given in Eq. (3) are chosen for the quantification of boom characteristics. In the optimization process, $\Delta P_{\max }$ is used as a boom optimization objective; PLdB is used as a constraint.

$$
\Delta P_{\max }=\max (\Delta P)-\min (\Delta P)
$$


The optimization for improved boom characteristics is carried out subject to the geometrical, stability, loudness, and aerodynamic constraints that are given in Eq. (4). These include geometrical constraints on both the fuselage $\left(D_{\text {fuselage }}\right)$ and the cabin diameter $\left(D_{\text {cabin }}\right)$, as well as on the maximum aft position of the wing's trailing edge $\left(X \max _{\text {wing }}\right)$ to prevent an unrealistic degree of sweep. Volumetric constraints are also placed on the fuselage $\left(V_{\text {fuselage }}\right)$ and the wing volume $\left(V_{\text {wing }}\right)$.

The design variables that are used in optimization include 24 shape function coefficients (A), as well as the fuselage diameter $\left(D_{\text {fuselage }}\right)$, the wing $x$-location $\left(X \operatorname{loc}_{\text {wing }}\right)$, and the root chord trailing edge location $\left(\delta_{\text {wing }}\right)$. The definition of the fuselage radius consists of six shape functions that are defined by 12 coefficients. The wing chord and sweep definitions each consist of three shape functions that are defined by six coefficients. All other vehicle definitions are kept fixed to the values of the baseline vehicle (see Fig. 8).

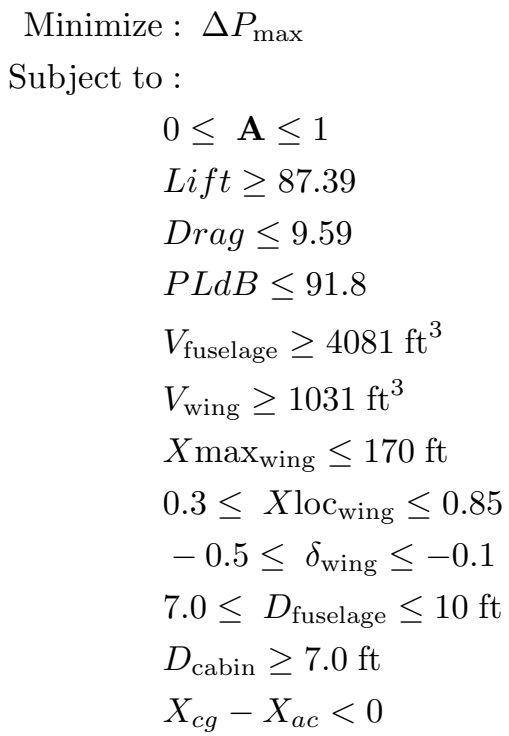

\section{Results}

\section{A. Fuselage-Wing Optimization}

The Cart3D CFD analyses were performed on a Linux cluster with 72 Intel Xeon E5462 at $2.8 \mathrm{GHz}$ processors. The optimization completed after 50 search steps and 649 function evaluations (iterations), which required a total run time of approximately 48 hours. The function evaluations, the convergence history for the objective function, and the optimum values for all of the design variables are provided in appendix B.

Remember that the optimization process that is demonstrated here does not utilize the baseline as a starting condition for the design variables. Design Explorer first conducts a design of experiments of 338 cases, as shown in appendix B, to sample the design space at orthogonal points and generate a Kriging-based surrogate model.

\section{Comparison of Optimum and Baseline Aircraft}

The resulting metrics of interest are shown in Table 2; the values for the baseline vehicle are included for comparison. The table shows two active constraints, including $X \max _{\text {wing }}$ and PLdB, as well as the improvement in $\Delta P_{\max }$, drag, and the lift-to-drag ratio of 15,6 , and 7.7 percent, respectively. In addition, the cabin radius and the fuselage and wing volumes have increased from those of the baseline. Note that the drag penalty due to an increase in wetted area is not present because Cart3D is an Euler CFD solver and viscous effects are not accounted for. 
Table 2. Fuselage-Wing Optimization Result and Comparison to Baseline.

\begin{tabular}{lrrrr}
\hline Metric & Baseline & Optimum & Units & Difference, \% \\
\hline \hline$X$ max $_{\text {wing }}$ & 170 & 170 & $\mathrm{ft}$ & 0 \\
$V_{\text {wing }}$ & 1031 & 1146 & $\mathrm{ft}^{3}$ & 11.1 \\
$V_{\text {fuselage }}$ & 4081 & 6010 & $\mathrm{ft}^{3}$ & 47.3 \\
$D_{\text {cabin }}$ & 7.0 & 8.5 & $\mathrm{ft}$ & 21.1 \\
$\mathrm{SM}$ & -33.5 & -38.2 & $\mathrm{ft}$ & 14.0 \\
Lift & 87.39 & 88.49 & - & 1.2 \\
Drag & 9.59 & 9.02 & - & -6.0 \\
Lift/Drag & 9.11 & 9.80 & - & 7.7 \\
PLdB & 91.8 & 91.8 & $\mathrm{PLdB}$ & 0 \\
Objective: $\Delta P_{\max }$ & 1.595 & 1.357 & $\mathrm{lb} / \mathrm{ft}^{2}$ & -15.0 \\
\hline
\end{tabular}

A top view of the baseline and the optimum design is shown in Fig. 9; the CFD pressure solutions are shown in Fig. 10 and 11. The distinct changes to the optimized design include the blunter nose shape with fuselage volume shifted toward the front part of the aircraft. These changes result from the lack of constraint on the initial shock pressure rise (SPR), so that the initial shock pressure rise is higher than that for the baseline vehicle. Note that the origin and the root chord of the optimum design are similar to those of the baseline. However, the optimized wing has a higher degree of sweep, and a chevron or kink exists in the chord below the nacelles.

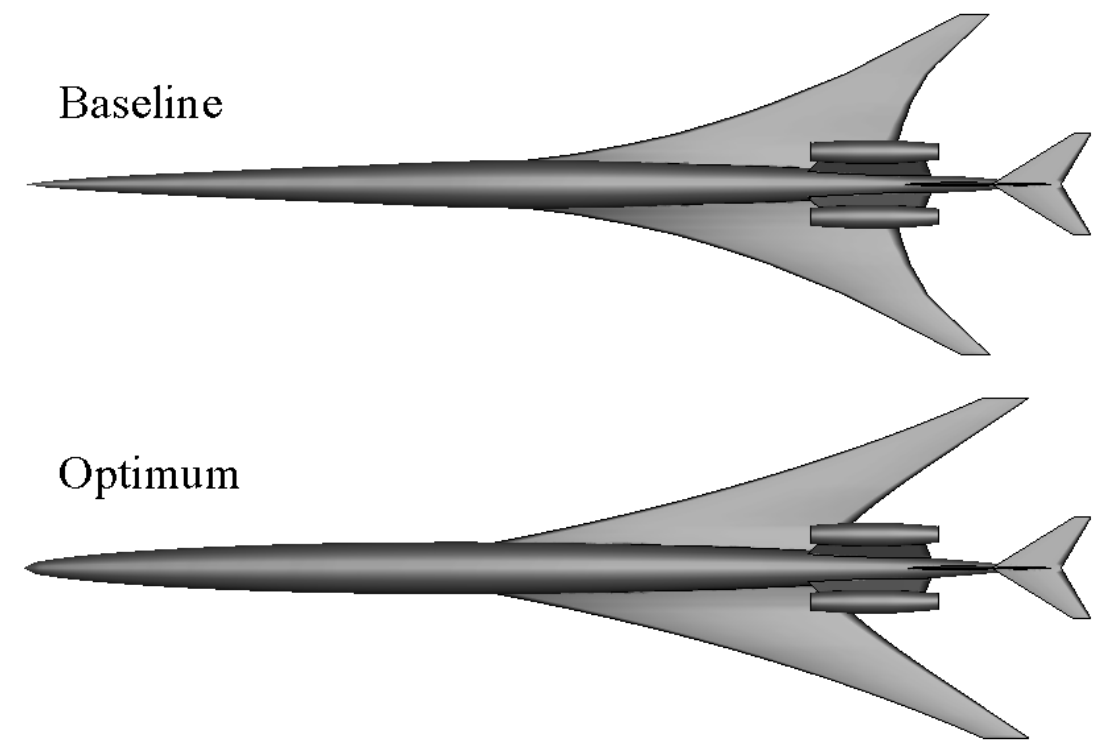

Figure 9. Comparison of geometry for fuselage-wing optimization.

The CFD pressure contours show a more smoothly shaped optimum fuselage as a result of the smooth behavior of the Kulfan analytical functions. A larger shock can also be identified at the nose of the optimized shape than can be found on the baseline. The initial shock is dispersed more effectively by the baseline aircraft because of the sharper, elongated nose. This results from the lack of a constraint on the initial shock pressure rise. The inclusion of this constraint in the optimization process likely shifts the volume that is required for the cabin aft in order to produce a more refined nose. Employing a larger number of shape functions to define of the fuselage radius may also yield a better optimized fuselage. Allowing more degrees of freedom can more easily facilitate the transition from the sharp nose to the required cabin radius within a shorter fuselage span. 


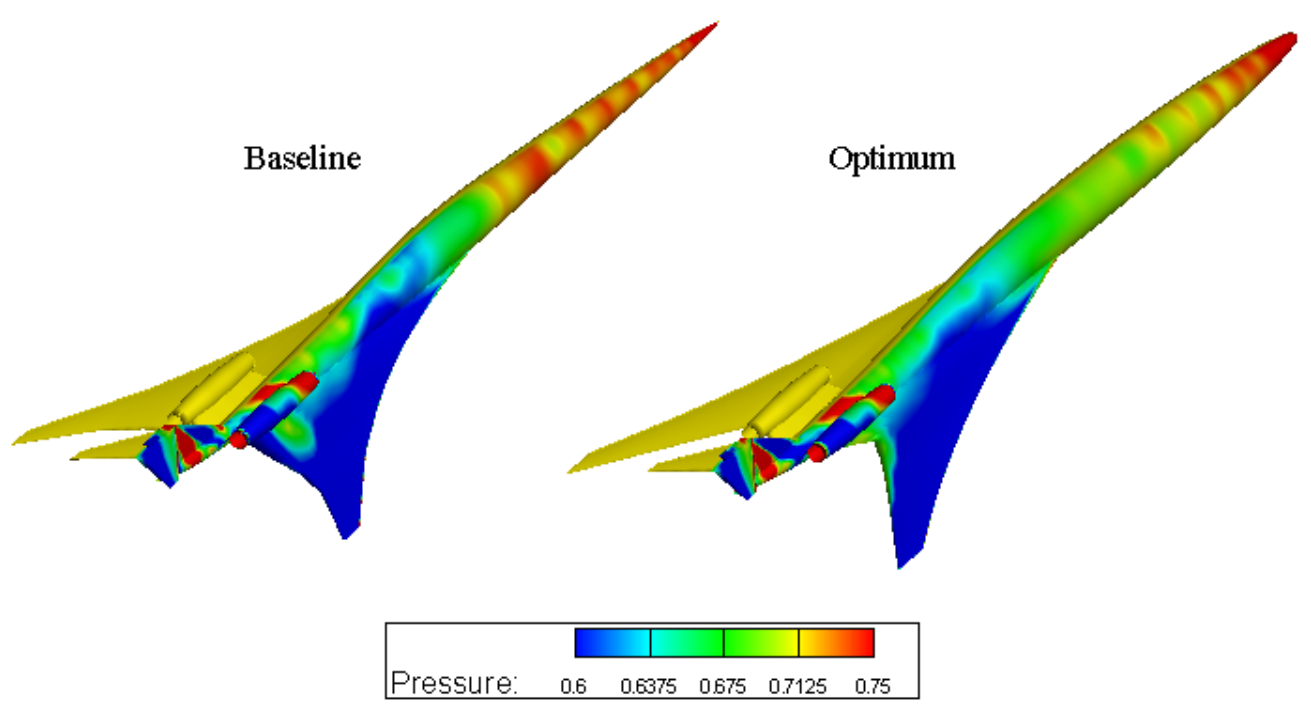

Figure 10. Comparison of CFD surface pressure contours for fuselage-wing optimization.

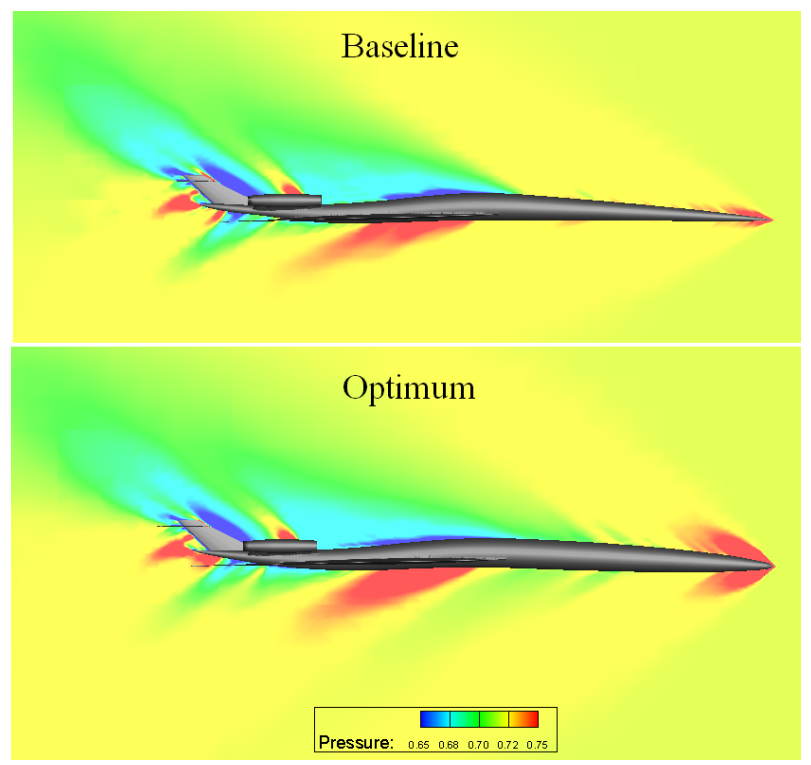

Figure 11. Comparison of CFD pressure contours at centerline for fuselage-wing optimization.

The volume, lift, and total equivalent areas for both the baseline and the optimum design are shown in Fig. 12. These were computed based on the CFD surface pressures shown in Fig. 10 and 11 . The increased fuselage volume is most likely a penalty on the degrees of freedom that were allowed for the fuselage radius definition. Due to scalability issues, the number of shape functions for the fuselage radius was limited to six with 12 coefficients (design variables). Despite the low number of shapes used, the total shape function count, including the chord and sweep grew to 24 design variables. The benefit to using the analytical shape function approach is that the problem can be easily scaled depending on the computational resources and the scope of the study. This scaling can be achieved by simply increasing the number of shape function coefficients for a given geometry definition while retaining a smooth behavior of the outer mold line. 


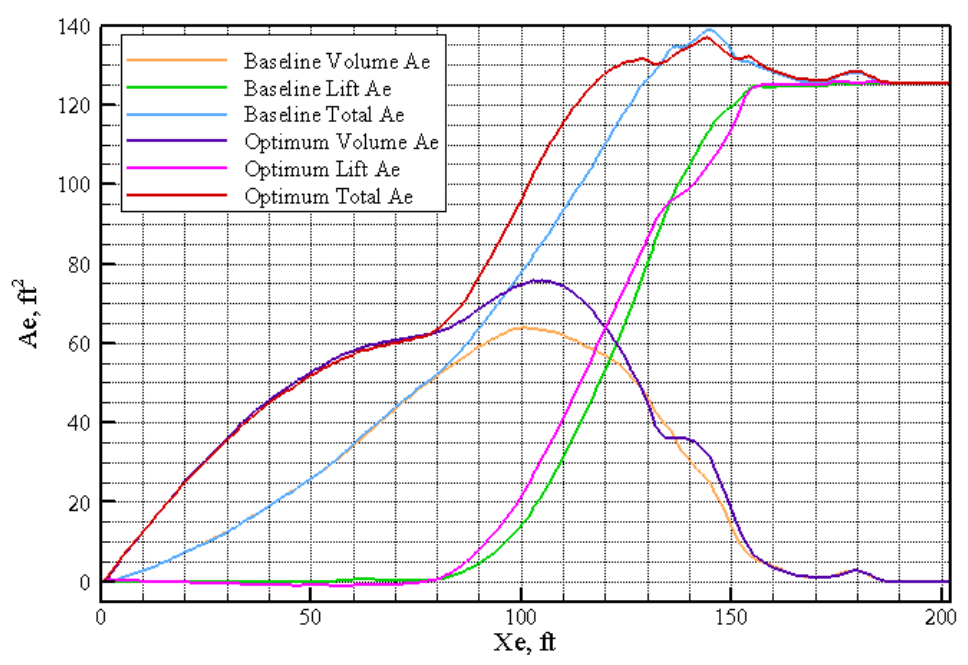

Figure 12. Equivalent area comparison for fuselage-wing optimization.

The ground signature comparison is shown in Fig. 13. This shows the rise in unconstrained initial shock pressure and two distinct larger shocks in the fore section with a slightly lower maximum overpressure. The true improvement in the signature occurred in the aft section of the aircraft, where a single large shock was diffused into three smaller shocks and the minimum overpressure was decreased by approximately $0.2 \mathrm{lb} / \mathrm{ft}^{2}$. This study introduced and successfully demonstrated the optimization process. Minimizing $\Delta P_{\max }$ by approximately $0.2 \mathrm{lb} / \mathrm{ft}^{2}$ while meeting or exceeding the volumetric, aerodynamic, and other boom metrics of the baseline demonstrates a successful boom mitigation process within the defined optimization objectives. However, the careful selection of the objectives is clearly key to designing a viable low-boom aircraft. The optimization process currently includes the capability to constrain or minimize the initial shock pressure rise; however, the capability to minimize a measure of error or deviation in order to match a target signature needs to be added in the future.

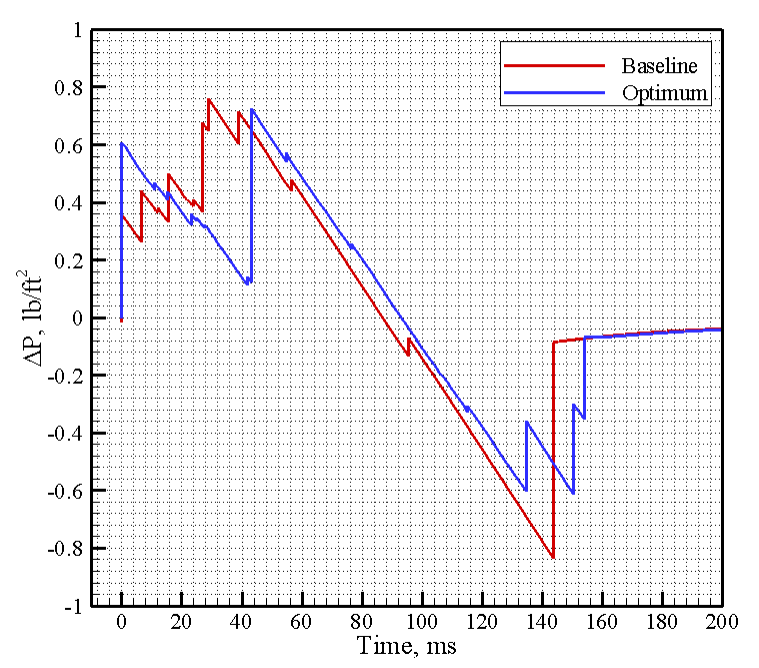

Figure 13. Ground signature comparison for fuselage-wing optimization.

\section{Design Space Exploration}

Design space exploration was conducted to assess the constraints and the variables that drive the design. The variable main effects for the optimization case are plotted in Fig. 14 . The variables A[0,1-11], $\mathrm{A}[1,1-6]$, and $\mathrm{A}[2,1-6]$ are shape function coefficients that correspond to the fuselage radius, wing chord, and sweep, respectively. The location of the wing has a significantly larger contribution to the objective 
$\left(\Delta P_{\max }\right)$ than the other design variables. Also note that although other individual design variables make only small contributions, their sum accounts for a large percentage of the objective. Another interesting result, which can be seen in the main effects plot, is that the overall contribution to the objective for the fuselage shaping coefficients $\mathrm{A}[0,1-11]$ is greater than that of the overall fuselage thickness. This result may be one reason why the optimization process improved the objective despite the fact that the fuselage volume was greater than that of the baseline.

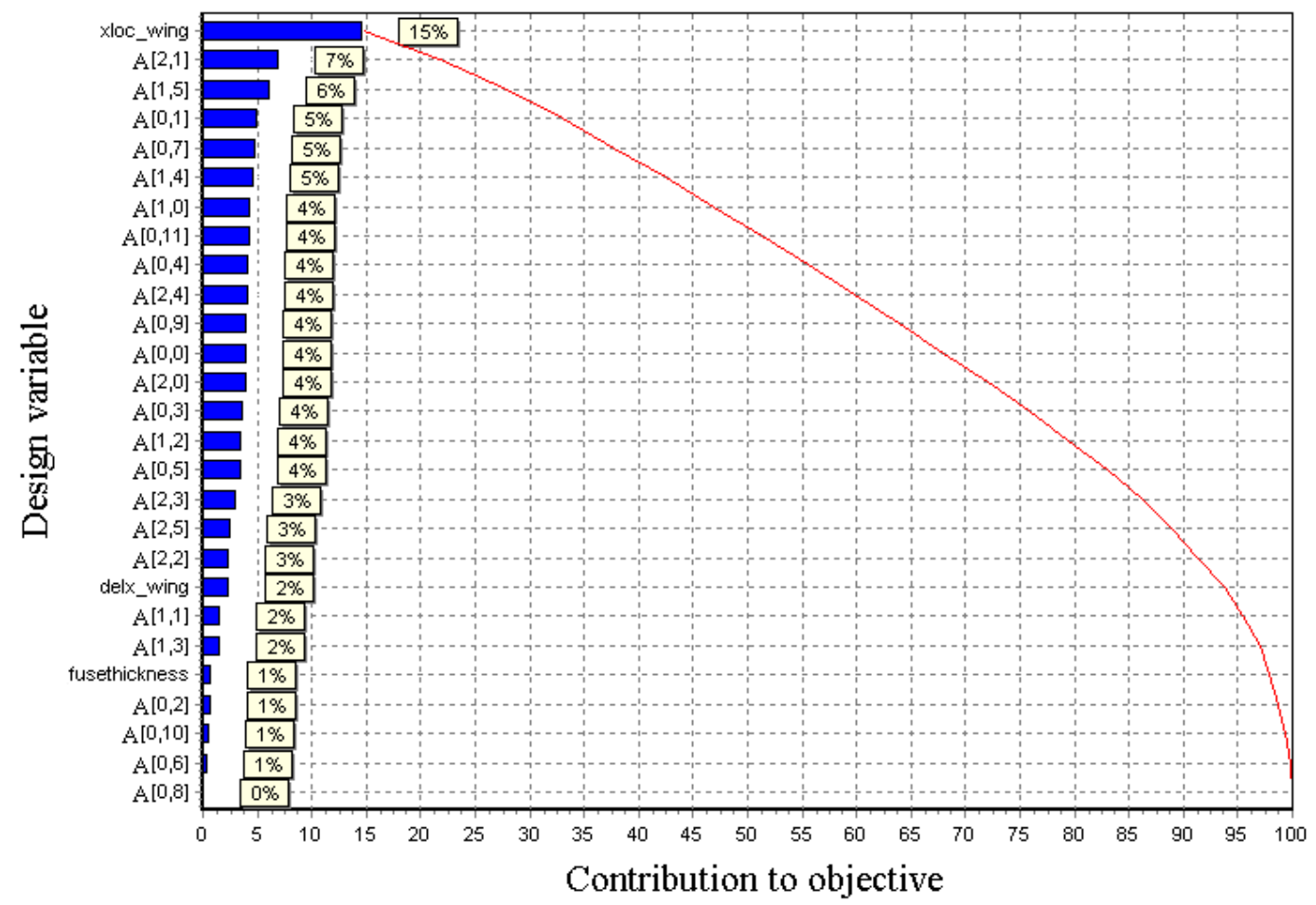

Figure 14. Design variable main effects for fuselage-wing optimization.

To better understand the key constraints that drive the design, contour plots were created for numerous pairwise sets of design variables. Figure 15 shows a contour plot for two design variables that correspond to the wing's leading edge sweep shape function coefficients. In the figure, the direction of improvement for the objective is indicated by the arrow, and the optimum design point is shown in black; thus, the feasible design space and two active constraints are evident. The first active constraint which

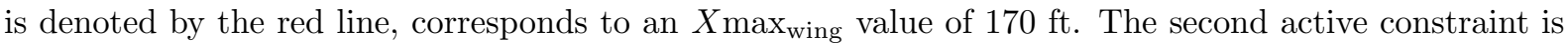
the PLdB value of 91.8. The improvement in drag is evidenced (see Table 2) by the fact that the design point moves away from the drag constraint (denoted by the magenta curve in Fig. 15) in the direction of improved drag characteristics. These two active constraints suggest two primary ways to further improve the objective. The first option is to increase the allowable wing sweep at the cost of structural feasibility. The second option is to push the PLdB constraint in the direction of improvement by decreasing the PLdB value; the PLdB metric is improved by decreasing the strength of the shocks and the frequency of occurrence with more refined control of the geometry. 


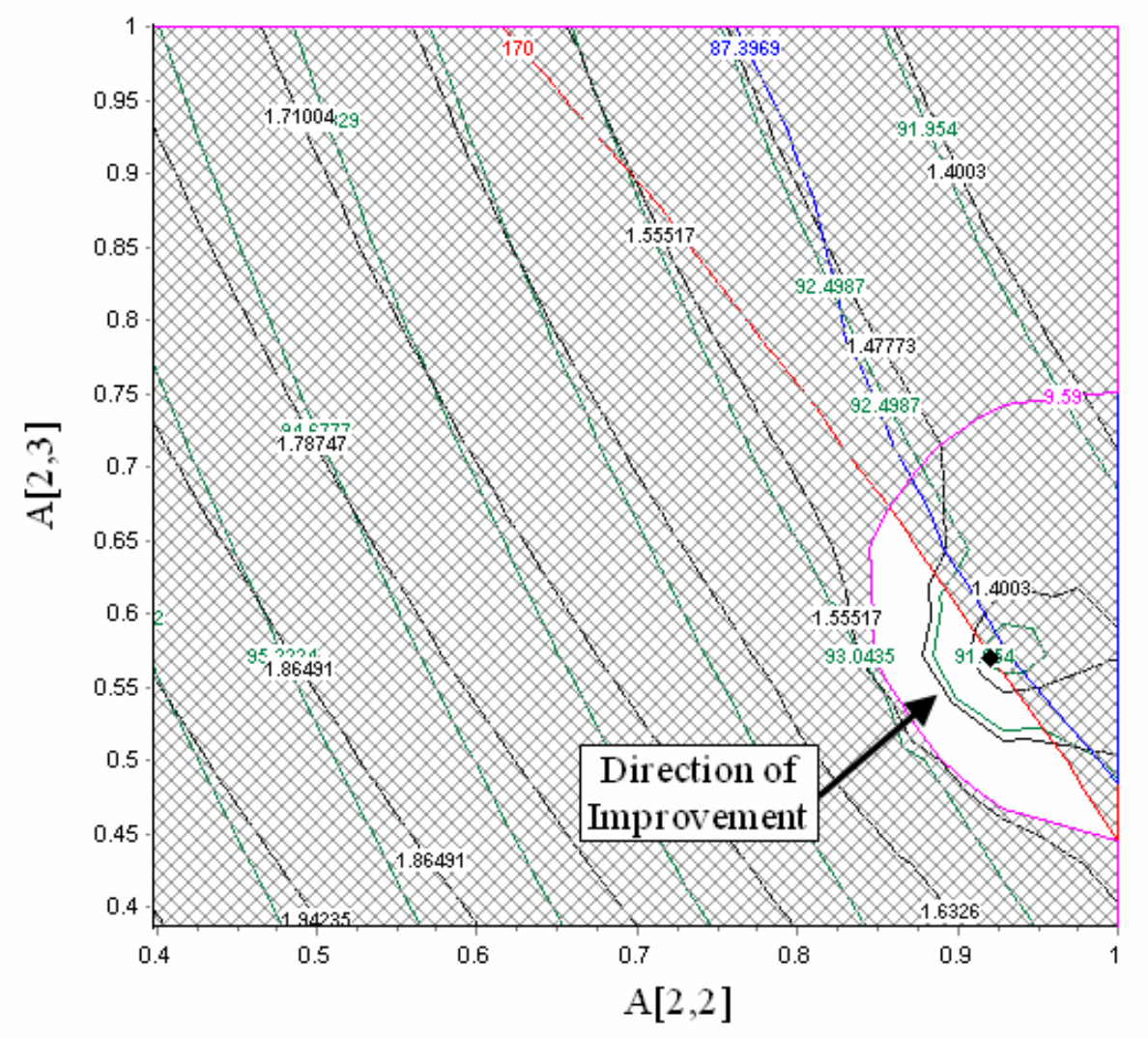

Figure 15. Contour plot of two coefficients corresponding to the wing sweep definition for fuselage-wing optimization.

\section{B. Wing Planform Optimization}

As seen earlier in the comparison of the fuselage-wing optimization results to the baseline, the initial shock pressure rise was not constrained and as a result the signature at the front part of the aircraft was degraded. The number of design variables used to describe the fuselage is also not sufficient and does not allow enough degrees of freedom to properly design the fuselage. The fuselage has a significant impact on the loudness level which restricts the feasible design space for the optimizer. A follow up optimization was conducted to verify this statement and assess whether the final design could be improved by excluding the fuselage and corresponding constraints from the optimization process.

A planform-only wing optimization was performed while holding all other geometric properties fixed to that of the baseline. The objective and all other constraints not pertaining to the fuselage from the previous fuselage-wing optimization also remain the same. The only geometry definitions that were allowed to vary included the wing chord, sweep, and $x$-location with respect to the fuselage. A total of 26 design variables were used, including 12 shape function coefficients for the wing chord, and sweep, respectively. Wing chord and sweep distributions each consisted of six shape functions including piecewise linear and nonlinear behaviors.

The results for the wing optimization case are shown in Table 3 and the final design variable settings are provided in appendix C. These show a significant improvement in maximum change in overpressure of 24.7 percent. In addition, the drag and loudness level have been indirectly reduced by 11.8 and 3.73 percent, respectively. 
Table 3. Wing Optimization Result and Comparison to Baseline.

\begin{tabular}{lrrrr}
\hline Metric & Baseline & Optimum & Units & Difference, \% \\
\hline \hline Xmax $_{\text {wing }}$ & 170 & 170 & $\mathrm{ft}$ & 0 \\
$V_{\text {wing }}$ & 1031 & 1031 & $\mathrm{ft}^{3}$ & 0 \\
SM & -33.5 & -35.2 & $\mathrm{ft}$ & 4.69 \\
Lift & 87.39 & 87.42 & - & 0.034 \\
Drag & 9.59 & 8.58 & - & -11.8 \\
Lift/Drag & 9.11 & 10.19 & - & 10.56 \\
PLdB & 91.8 & 88.5 & $\mathrm{PLdB}^{2}$ & -3.73 \\
Objective: $\Delta P_{\max }$ & 1.595 & 1.275 & $\mathrm{lb} / \mathrm{ft}^{2}$ & -24.7 \\
\hline
\end{tabular}

The optimum wing planform is compared to the baseline in Fig. 16. The trailing edge shows a greater nonlinear behavior because the increase in the number of shape functions has introduced more nonlinear degrees of freedom. Similarly, the pressure solution for the wing optimization case is shown in Fig. 17.

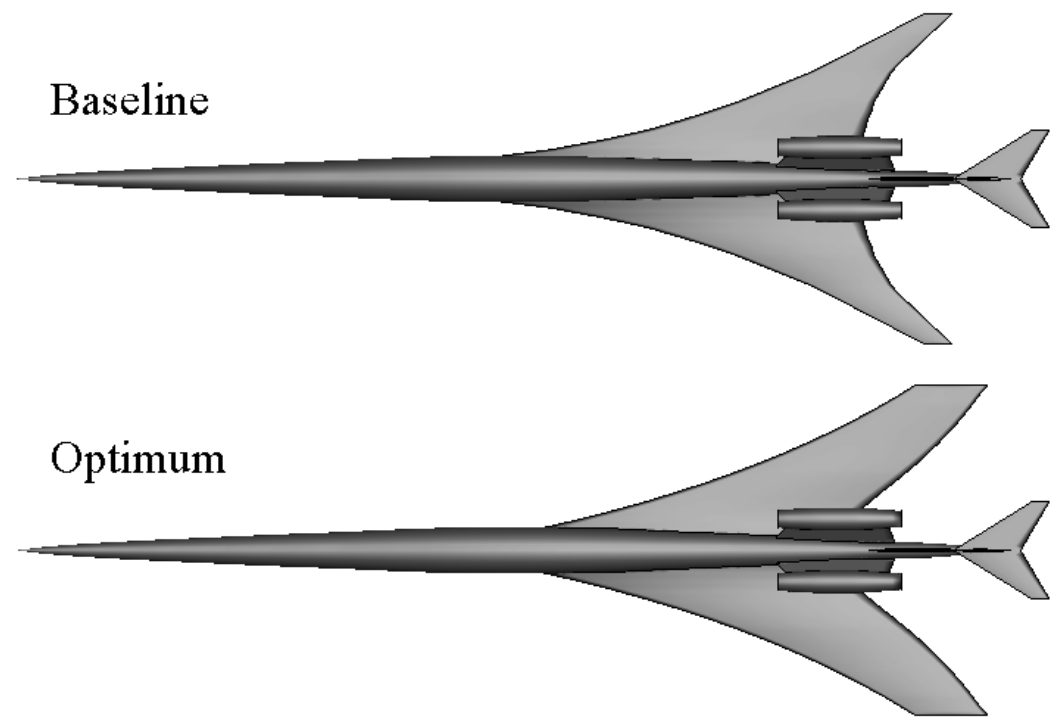

Figure 16. Comparison of geometry for wing optimization.
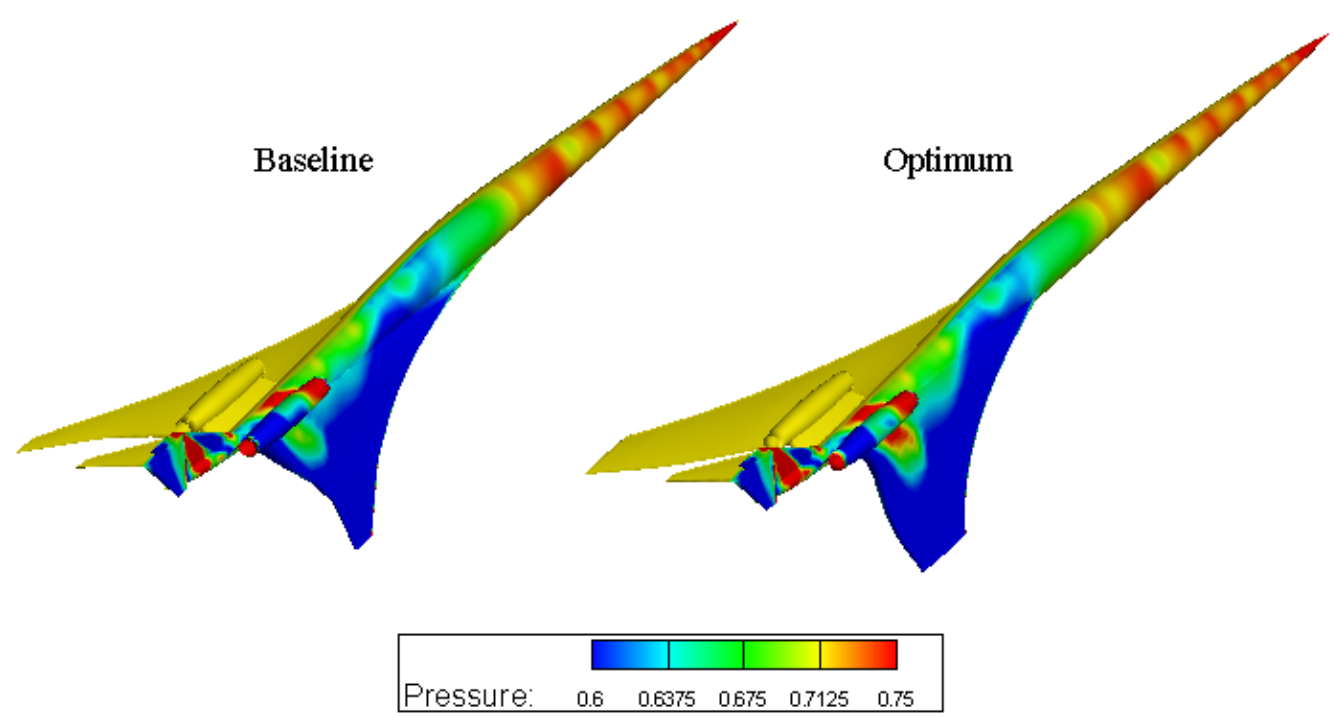

Figure 17. Comparison of CFD surface pressure contours for wing optimization. 
The equivalent area comparison is provided in Fig. 18. As expected, the area in the front portion of the aircraft remains unchanged since the fuselage was fixed to that of the baseline. The optimizer has flattened the volume equivalent area by shifting some of the wing volume to the aft part of the aircraft. Similarly, the lift equivalent area has been shifted back in comparison to the baseline.

The ground signature plot is shown in Fig. 19. This wing optimized signature shows a significant improvement in $\Delta P_{\max }$ while maintaining the well behaved shocks in the front portion of the aircraft. In addition, the minimum overpressure in the aft part of the signature has been improved and the aft shock has been split into three smaller shocks. The optimization has been successful because not only have the objective and aerodynamic efficiency been improved but the final signature falls inside the envelope of the baseline signature without any localized degradation.

The wing optimization presented demonstrates that the original fuselage discretization in the previous optimization case was not sufficient. The lack of sufficient fuselage degrees of freedom was limiting the optimizer's ability to find a feasible loudness domain. Additional work is still needed to determine the adequate number of design parameters required to properly shape the fuselage.

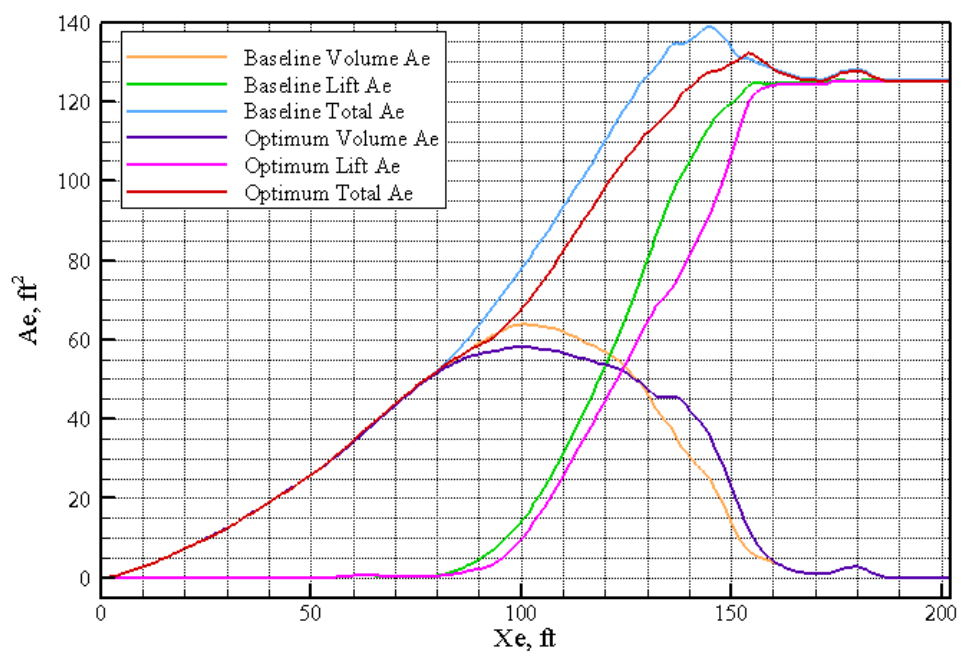

Figure 18. Equivalent area comparison for wing optimization.

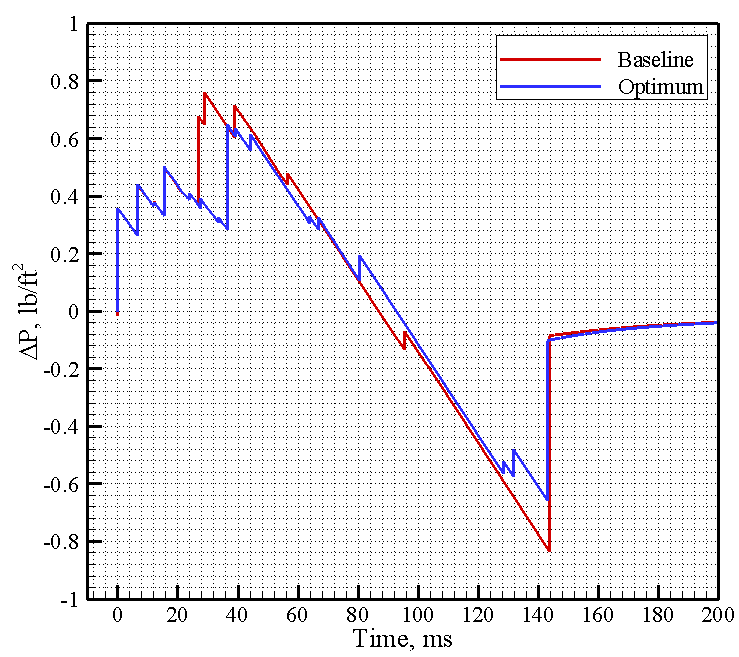

Figure 19. Ground signature comparison for wing optimization. 


\section{Concluding Remarks}

A parametric framework has been developed for the design and optimization of supersonic aircraft with the use of analytical shape functions. The framework is designed to be modular and flexible in the sense that additional aircraft components and greater level of optimization complexity can be easily selected for a design optimization or study. The Analytical Shaping Tool (AST) is capable of shaping fuselage, wing, canard, tail, fin, pylon, and nacelle components. In addition, the parameters that define the location and orientation of these components can be manipulated and easily incorporated into the optimization process.

A great deal of work went into integrating and automating the high-fidelity computational fluid dynamics (CFD) analysis. The use of more sophisticated CFD opens the door for the analysis of more complex and novel configurations that have not been able to be properly analyzed with low-fidelity tools. The ability to examine more advanced aircraft concepts potentially opens the design space to better design solutions.

The boom analysis that is currently in the framework is based on a mixed-fidelity approach which consists of CFD pressure distributions coupled with low-fidelity equivalent area methods. This is a compromise that took place in order to make the fully automated optimization process feasible. Ultimately, the framework can be expanded to high-fidelity analysis by eliminating the simplified equivalent area theory once improved numerical optimizers have been explored and the parameterization and computational resources become more efficient.

A fuselage-wing optimization was presented to demonstrate a subset of the capabilities that have been built into the framework. The optimization successfully demonstrated that optimization based on analytical shape functions is possible. The objective of maximum change in overpressure $\left(\Delta P_{\max }\right)$ was decreased by approximately 15 percent while the other aerodynamic, geometrical, and stability requirements were met or exceeded. However, the selection of proper boom metric objectives and discretization scheme was clearly shown to be key to successful design of a low-boom aircraft. In the fuselage-wing optimization, the optimizer improved $\Delta P_{\max }$ at the cost of a larger SPR. The placement of constraints or objectives on the SPR, the maximum and minimum overpressure, the total impulse, and the target signature matching are possible solutions to ensure that a desired signature is obtained. The later wing optimization showed that the discretization scheme for the fuselage was limiting the degrees of freedom and feasible domain of the optimizer. It demonstrated a significant improvement in boom and aerodynamic characteristics of 24.7 and 11.8 percent, respectively, without any localized degradation of the ground signature.

\section{Future Work}

The following is a list of tasks that still must be carried out to further improve the framework's analysis and system studies capabilities.

- Incorporate a capability to perform target-based optimization of a pressure signature.

- Create capability for back solving shape function coefficients from an existing VSP geometry. This will allow deformation from a starting geometrical definition with the use of a gradient-based optimization.

- Assess variable parameterization and discretization requirements in optimization for a more robust design space exploration.

- Explore other optimization approaches and assess the advantages and disadvantages with the hope of improving design space exploration.

- Add circular wing and V-tail component capabilities to AST for the study and optimization of more advanced and novel concepts.

- Incorporate CFD-based engine simulation in the Cart3D analysis for a more accurate prediction of sonic boom characteristics. 
Appendix A. Grid Sensitivity Study

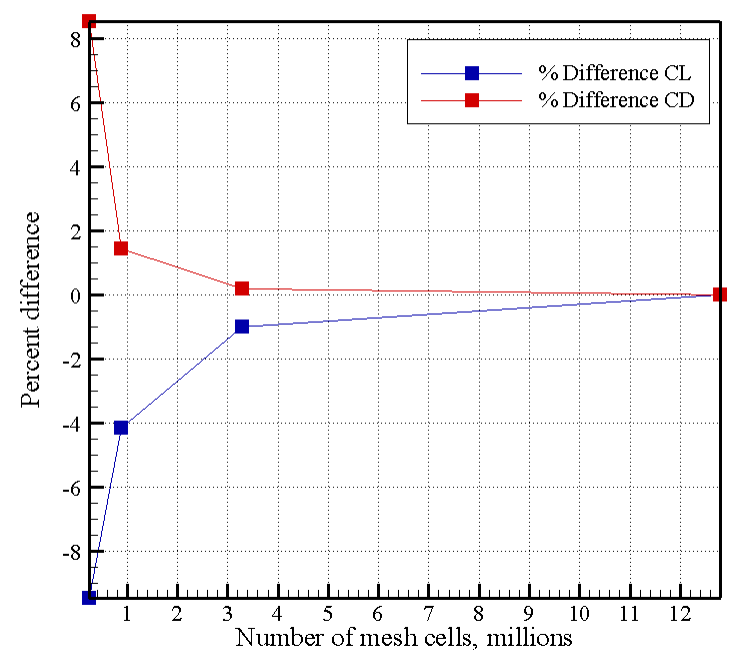

Figure A1. Lift and drag sensitivity to grid size.

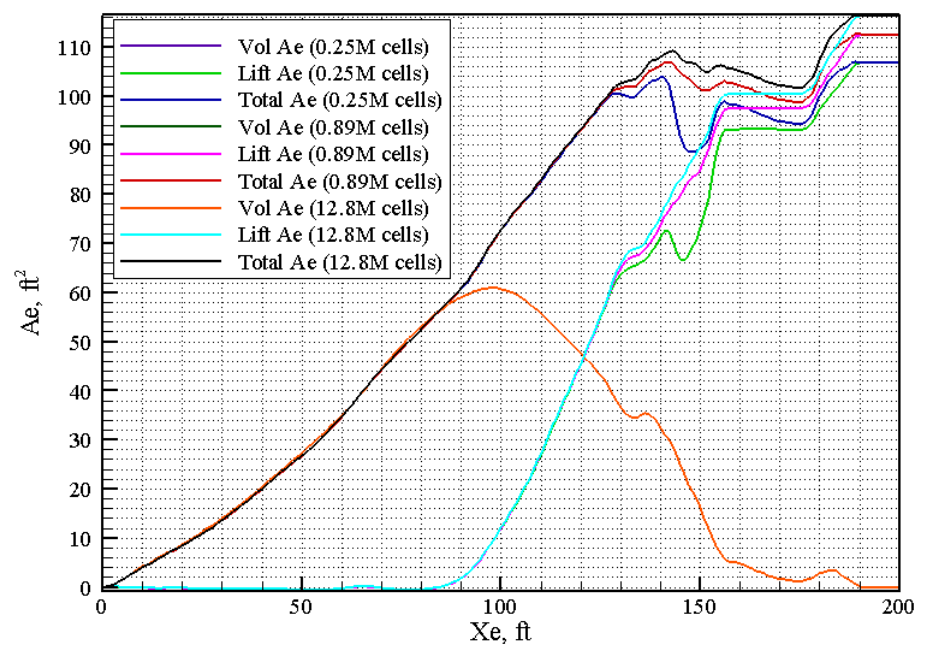

Figure A2. Total equivalent area sensitivity to grid size. 


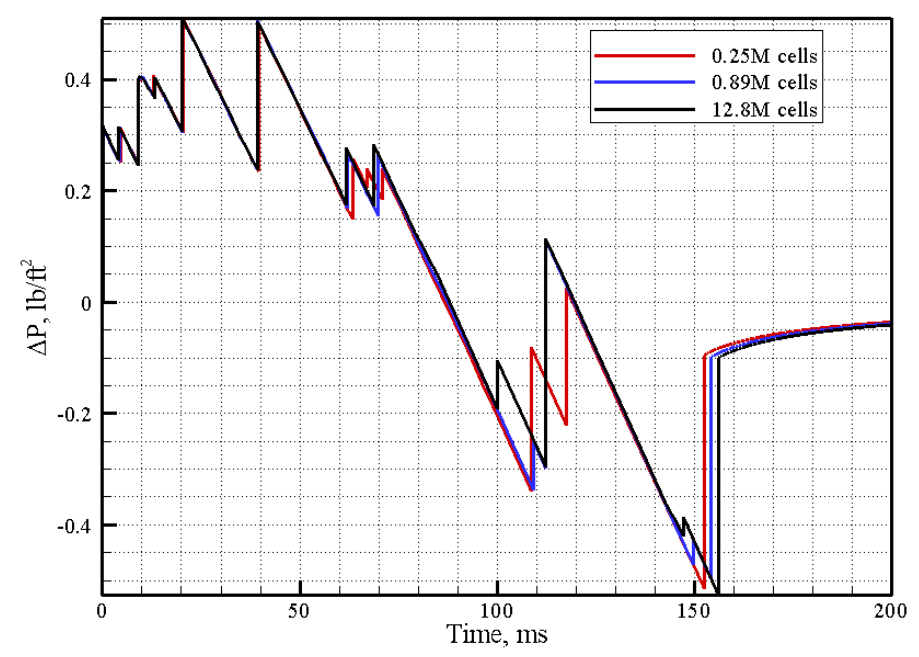

Figure A3. Signature sensitivity to grid size.

Appendix B. Convergence and Variable Settings for Fuselage-Wing Optimization

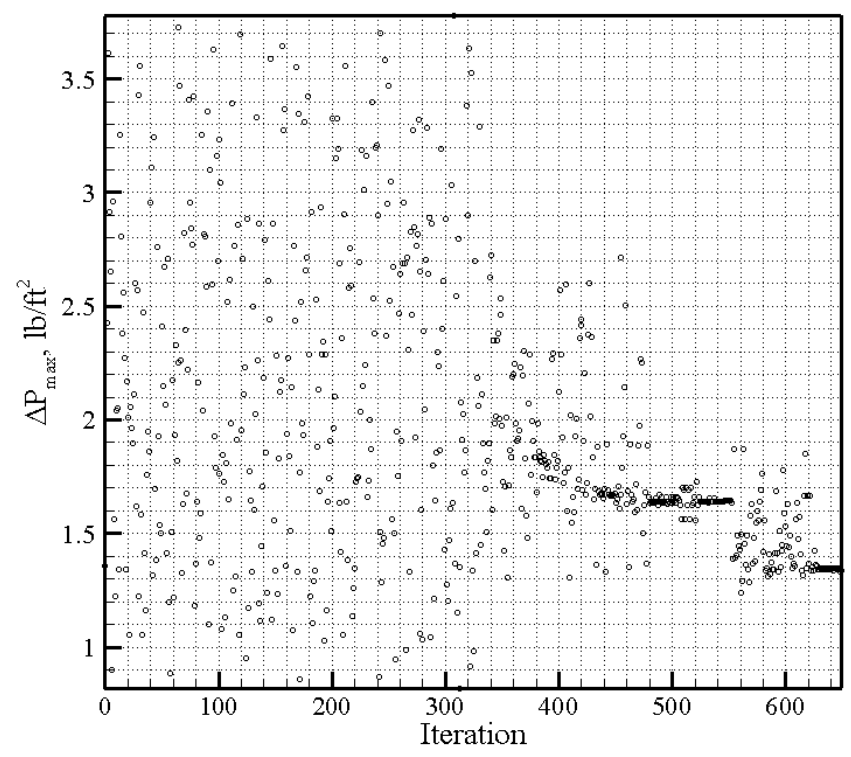

Figure B1. Raw function evaluations of objective. 


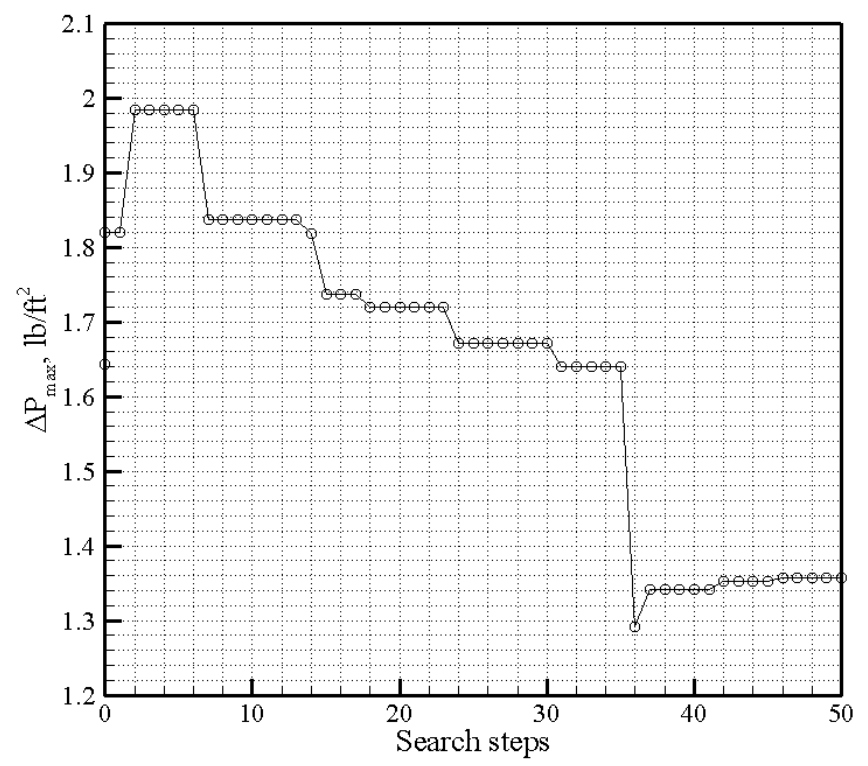

Figure B2. Objective convergence history.

Table B1. Optimum Shape Function Coefficients for Fuselage-Wing Optimization (A).

\begin{tabular}{|l|c|c|c|c|r|r|}
\hline Design variable & Coeff 1 & Coeff 2 & Coeff 3 & Coeff 4 & Coeff 5 & Coeff 6 \\
\hline \hline Fuselage radius & 1.0000 & 0.8594 & 0.3965 & 0.7637 & 1.0000 & 1.0000 \\
\hline Wing chord & 0.0137 & 1 & 0.3086 & 0.2168 & 1.0000 & 1.0000 \\
\hline Wing sweep & 0.9336 & 1.0000 & 0.9277 & 0.5684 & 0.8300 & 0.0000 \\
\hline
\end{tabular}

\begin{tabular}{|l|c|c|c|c|c|c|}
\hline Design variable & Coeff 7 & Coeff 8 & Coeff 9 & Coeff 10 & Coeff 11 & Coeff 12 \\
\hline \hline Fuselage radius & 0.7637 & 1.0000 & 0.5898 & 1.0000 & 0.4063 & 0.9629 \\
\hline Wing chord & - & - & - & - & - & - \\
\hline Wing sweep & - & - & - & - & - & - \\
\hline
\end{tabular}

Table B2. Optimum Design Variables for Fuselage-Wing Optimization.

\begin{tabular}{|l|c|}
\hline Design variable & Value \\
\hline \hline$D_{\text {fuselage }}$ & $8.833 \mathrm{ft}$ \\
\hline$X$ loc $_{\text {wing }}$ & 0.320 \\
\hline$\delta_{\text {wing }}$ & -0.176 \\
\hline
\end{tabular}




\section{Appendix C. Variable Settings for Wing Optimization}

Table C1. Optimum Shape Function Coefficients for Wing Optimization (A).

\begin{tabular}{|l|c|c|c|c|c|r|}
\hline Design variable & Coeff 1 & Coeff 2 & Coeff 3 & Coeff 4 & Coeff 5 & Coeff 6 \\
\hline \hline Wing chord & 0.0000 & 0.9785 & 0.0020 & 1.0000 & 1.0000 & 0.9941 \\
\hline Wing sweep & 0.9727 & 0.8887 & 0.9395 & 0.5859 & 0.7617 & 0.0000 \\
\hline
\end{tabular}

\begin{tabular}{|l|r|r|r|r|r|r|}
\hline Design variable & Coeff 7 & Coeff 8 & Coeff 9 & Coeff 10 & Coeff 11 & Coeff 12 \\
\hline \hline Wing chord & 0.9941 & 0.1934 & 0.5449 & 0.9707 & 0.0059 & 0.7617 \\
\hline Wing sweep & 0.9981 & 0.3398 & 0.6055 & 0.0000 & 0.9512 & 0.0000 \\
\hline
\end{tabular}

Table C2. Optimum Design Variables for Wing Optimization.

\begin{tabular}{|l|c|}
\hline Design variable & Value \\
\hline \hline$X$ loc $_{\text {wing }}$ & 0.4203 \\
\hline$\delta_{\text {wing }}$ & -0.1625 \\
\hline
\end{tabular}

\section{Acknowledgements}

The authors would like to thank all members of the Systems Integration, Assessment and Validation group at NASA LaRC, particularly, Wu Li, Sharon L. Padula, Karl A. Geiselhart, James W. Fenbert, Elwood W. Shields, and Lori P. Ozoroski. Their help, experience, and expertise was vital to the work presented here.

\section{References}

1 "Model Center and Optimization Tools," Phoenix Integration, http://www.phoenix-int.com/, Dec. 2009.

${ }^{2}$ Kulfan, B. M. and Bussoletti, J. E., "Fundamental Parametric Geometry Representations for Aircraft Component Shapes," .

${ }^{3}$ Lores., M. E., Burdges, K. P., and Shrewsbury, G. D., "Analysis of a Theoretically Optimized Transonic Airfoil," NASA CR-3065, 1978. 2009.

${ }^{4}$ Aftosmis, M., "Cart3D Resource Website," http://people.nas.nasa.gov/ aftosmis/cart3d/cart3Dhome.html, Dec.

${ }^{5}$ Gloudemans, J. R., Davis, P. C., , and Gelhausen, P. A., "A Rapid Geometry Modeler for Conceptual Aircraft," AIAA 96-0052, 1996.

${ }^{6}$ Seebass, R., "Sonic Boom Theory," Journal of Aircraft, Vol. 6, No. 3, May 1969, pp. 177-184.

${ }^{7}$ Maglieri, D. J., Carlson, H. W., and Hubbard, H. H., "Status of Knowledge of Sonic Booms," NASA TM-80113, 1979 .

${ }^{8}$ Seebass, R. and George, A., "Sonic Boom Minimization," Journal of Acoustical Society of America, Vol. 51, No. 2, 1972, pp. 686-694.

${ }^{9}$ Darden, C., "Sonic Boom Minimization with Nose-Bluntness Relaxation," NASA TP-1348, Sept. 1979.

${ }^{10}$ Mack, R. J., "A Supersonic Business-Jet Concept Designed for Low Sonic Boom," NASA TM-2003-212435, Oct. 2003 .

${ }^{11}$ Rallabhandi, S. K. and Mavris, D. N., "Aircraft Geometry Design and Optimization for Sonic Boom Reduction," AIAA Journal of Aircraft, Vol. 44, No. 1, 2007, pp. 35-47.

${ }^{12}$ Howe, D. C., Waithe, K. A., and Haering, E. A., "Quiet Spike Near Field Flight Test Pressure Measurements with Computational Fluid Dynamics Comparisons," AIAA 2008-128, 2008.

${ }^{13}$ Page, J. A. and Plotkin, K. J., "An Efficient Method for Incorporating Computational Fluid Dynamics into Sonic Boom Prediction," AIAA 91-3275, 1991.

${ }^{14}$ Plotkin, K. J. and Page, J. A., "Extrapolation of Sonic Boom Signatures from CFD Solutions," AIAA 2002-922, 2002.

${ }^{15}$ Rallabhandi, S. K. and Mavris, D. N., "New Computational Procedure for Incorporating Computational Fluid Dynamics into Sonic Boom Prediction," AIAA Journal of Aircraft, Vol. 44, No. 6, 2007, pp. $1964-1971$.

${ }^{16}$ Coen, P. G., "Development of a Computer Technique for the Prediction of Transport Aircraft Flight Profile Sonic Boom Signatures," Master's Thesis, George Washington University, March 1983.

${ }^{17}$ Wallace, H. D., Haefeli, R. C., and Kulsrud, H. E., "Sonic Boom Propagation in a Stratified Atmosphere with Computer Program," NASA CR-1299, April 1969.

${ }^{18}$ Plotkin, K. J., "PCBoom3 Sonic Boom Prediction Model - Version 1.0c," Wyle Research Laboratories AFRL-HEWP-TR-2001-0155, Arlington, VA, May 1996. 
${ }^{19}$ Audet, C., Dennis, J., Moore, D. W., Booker, A., and Frank, P. D., "A Surrogate-Model-Based Method for Constrained Optimization," AIAA 2000-4891, Sept. 2000.

${ }^{20}$ Currin, C., Mitchell, T., Morris, M., and Ylvisaker, D., "A Bayesian Approach to the Design and Analysis of Computer Experiments," Oak Ridge National Laboratory Technical Report ORNL-6498, 1988.

${ }^{21}$ Currin, C., Mitchell, T., Morris, M., and Ylvisaker, D., "Bayesian Prediction of Deterministic Functions, with Applications to the Design and Analysis of Computer Experiments," Journal of the American Statistical Association, 1991, pp. 953-963.

${ }^{22}$ Shepherd, K. and Sullivan, B., "A Loudness Calculation Procedure Applied to Shaped Sonic Booms," NASA TP-3134, Nov. 1991. 